\title{
Global diffeomorphism of the Lagrangian flow-map for Pollard-like solutions
}

\author{
Adrián Rodríguez-Sanjurjo ${ }^{1}$
}

Received: 12 February 2018 / Accepted: 16 April 2018 / Published online: 27 April 2018

(C) Fondazione Annali di Matematica Pura ed Applicata and Springer-Verlag GmbH Germany, part of Springer Nature 2018

\begin{abstract}
We present a rigorous analysis of the nonlinear surface waves in the presence of a zonal current under the effects of Earth's rotation derived by Constantin and Monismith ( $\mathrm{J}$ Fluid Mech 820:511-528, 2017). It is shown that the three-dimensional Lagrangian flow-map describing this exact solution is a global diffeomorphism, resulting in a flow description that is dynamically possible.
\end{abstract}

Keywords Global diffeomorphism · Geophysical water waves · Exact and explicit solution

Mathematics Subject Classification $35 \mathrm{Q} 86 \cdot 35 \mathrm{~A} 16 \cdot 35 \mathrm{C} 05 \cdot 35 \mathrm{Q} 35$

\section{Introduction}

The solution derived by Gerstner [12] and rediscovered by Froude [11], Rankine [26] and Reech [27] provides the only known explicit and exact solution of the nonlinear periodic gravity wave problem with a non-flat surface. Despite being a remarkable achievement, the fact that the flow described for such a solution is rotational and cannot be generated by conservative forces made the development of Gerstner's theory much less widespread than the study of Stokes waves. Nevertheless, Gerstner waves constitute more than an interesting mathematical achievement. Laboratory experiments such as [23] insinuated the existence of Gerstner waves under a mean Eulerian flow which neutralises the Stokes drift (which is the lowest-order observable difference between Gerstner and higher-order Stokes waves). Accordingly, the study of these type of waves and its different applications has been growing lately.

The first rigorous analysis of Gerstner wave was performed by Constantin [3] and subsequently by Henry [13], setting the appropriate mathematical framework for its understanding.

Adrián Rodríguez-Sanjurjo

a.rodriguezsanjurjo@umail.ucc.ie

1 School of Mathematical Sciences, University College Cork, Cork, Ireland 
Gerstner-like solutions describing a diversity of geophysical models have appeared over the last years. In particular, [6] presents a geophysical generalisation of Gerstner wave, in the sense that the former is recovered by ignoring the Coriolis terms. This was further extended to include several physical scenarios including equatorially trapped and internal waves [4,6$8,19,20,22,24,31]$ as well as currents [14-16]; for the interested reader a review of these topics is available in [17] and [21]. Prior to this work, Pollard [25] extended Gerstner's description to an incompressible vertically stratified fluid in a rotating system. In common with Gerstner's solution, the particle paths are circular; however, they lie in a plane slightly tilted in a certain angle dependent on the Earth's latitude. Recently, Constantin and Monismith [9] performed a Lagrangian analysis of the nonlinear Pollard's surface waves with the additional complexity of incorporating a zonal current while maintaining the effects of the Earth's rotation. The incorporation of the current is a remarkable achievement and it does not only allow the generalisation of waves obtained by Pollard but it also provides a new type of wave motion which is characterised by waves with small vertical scales for gravity wave wavelengths and such that in the case of a much longer wavelength it produces waves with vertical decay scales of metres.

The aim of this paper is to provide a rigorous mathematical analysis of the exact solution derived in [9] and given by (2.2), proving that such solution is dynamically possible. Although the compatibility of this solution with the governing equations in the $f$-plane approximation has been already proven, the fact that the map describing the Lagrangian description is a global diffeomorphism remained unproven. This last property assures that the Lagrangian map (2.2) is a purely three-dimensional motion of the whole fluid body where all the particles describe inertial circles slightly tilted with respect to the vertical and which radius at each latitude decreases with depth; furthermore, the particles do not collide and they completely fill the infinite water region below the free surface.

\section{The Pollard solution}

\subsection{The governing equations}

The fluid motion considered in this paper describes surface water waves propagating zonally and with a wavelength such that the effects of the Earth's rotation are significant. Consequently, the Coriolis forces must be taken into account, leading to additional terms in the equations of motion. For the purposes of the present study, the Earth is taken as a perfect sphere of radius $R=6378 \mathrm{~km}$, rotating with constant rotational speed of $\Omega=7.29 \times 10^{-5} \mathrm{rad} \mathrm{s}^{-1}$ around the polar axis towards east. Subsequently, the choice of a rotating framework is made, where the origin is taken at a point on Earth's surface, the zonal coordinate $x$ is pointing horizontally due east, the meridional coordinate $y$ is pointing horizontally due north, whereas the coordinate $z$ corresponds to the local vertical and it is oriented "upward" (in the direction of the line joining the centre of the Earth with the point of the surface taken as origin). The governing equations for geophysical ocean waves follow from the Euler equations for this particular setting (cf. [10]). Therefore, for the velocity field $(u, v, w)$, we have that

$$
\left\{\begin{array}{l}
u_{t}+u u_{x}+v u_{y}+w u_{z}+2 \Omega w \cos \phi-2 \Omega v \sin \phi=-\frac{1}{\rho} P_{x}, \\
v_{t}+u v_{x}+v v_{y}+w v_{z}+2 \Omega u \sin \phi=-\frac{1}{\rho} P_{y}, \\
w_{t}+u w_{x}+v w_{y}+w w_{z}-2 \Omega u \cos \phi=-\frac{1}{\rho} P_{z}-g,
\end{array}\right.
$$


where $\phi$ represents the latitude, $g=9.8 \mathrm{~ms}^{2}$ is the gravitational constant, $\rho$ represents the water density and $P$ is the pressure. We note that the Coriolis parameters $f=2 \Omega \sin \phi$ and $\hat{f}=2 \Omega \cos \phi$ have been made explicit. In particular, we are interested in waves propagating zonally in a narrow ocean strip, so the choice of $f$ and $\hat{f}$ as constants is justified, leading to the so-called $f$-plane approximation of the Euler equations,

$$
\left\{\begin{array}{l}
u_{t}+u u_{x}+v u_{y}+w u_{z}+\hat{f} w-f v=-\frac{1}{\rho} P_{x}, \\
v_{t}+u v_{x}+v v_{y}+w v_{z}+f u=-\frac{1}{\rho} P_{y}, \\
w_{t}+u w_{x}+v w_{y}+w w_{z}-\hat{f} u=-\frac{1}{\rho} P_{z}-g .
\end{array}\right.
$$

However, the constant values of $f$ and $\hat{f}$ will depend upon the latitude; for instance, the Coriolis parameter $f$ vanishes along the equator, and $f=\hat{f} \approx 10^{-4} \mathrm{~s}^{-1}$ for a latitude $45^{\circ}$ North. In addition, (2.1a) is coupled with the equation of mass conservation

$$
\frac{D \rho}{D t}=0
$$

together with

$$
\nabla \cdot \vec{u}=0,
$$

where $\nabla \cdot \vec{u}$ expresses the compression rate of the fluid (see [5] for more details) and $D / D t$ represents here the material or convective derivative. Furthermore, boundary conditions for deep water waves need to be included. The dynamic boundary condition being

$$
P=P_{\mathrm{atm}} \text { at the free surface } z=\eta(x, y, t),
$$

where $P_{\text {atm }}$ is taken to be the constant atmospheric pressure at the surface of the ocean. On the other hand, the required kinematic boundary condition is

$$
w=\frac{D \eta}{D t} \text { on the free surface } z=\eta(x, y, t),
$$

which can be interpreted as the fact that a fluid particle located initially at the free surface must remain there for all time. Finally, the fluid motion at the bottom of the ocean is taken to be negligible.

\subsection{Solution}

The solution derived by Constantin and Monismith [9] is given by means of the Lagrangian framework (see [2] for a detailed analysis of the Lagrangian approach). As it was proven in [9], it constitutes an exact and explicit solution to the governing equations (2.1) for nonlinear waves propagating zonally on a zonal current $c_{0}$ and under the effects of Earth's rotation. The position at each time $t$ of a particle labelled by $(q, r, s)$ is given by

$$
\left\{\begin{array}{l}
x=q-c_{0} t-b e^{m s} \sin [k(q-c t)] \\
y=r-d e^{m s} \cos [k(q-c t)] \\
z=s+a e^{m s} \cos [k(q-c t)],
\end{array}\right.
$$

where the label $(q, r, s)$ should not be confused with the initial position of a particle and whose domain of definition is the set $\mathbb{R} \times\left(-r_{0}, r_{0}\right) \times\left(-\infty, s_{0}(r)\right)$, for a given $r_{0}$ and $s_{0}(r)<0$. (see Fig. 1 for two examples of the trajectories of the fluid particles). On the other hand, the real parameters $a, b, d, m$, as well as the wavenumber $k$ and the wave speed $c$ satisfy 


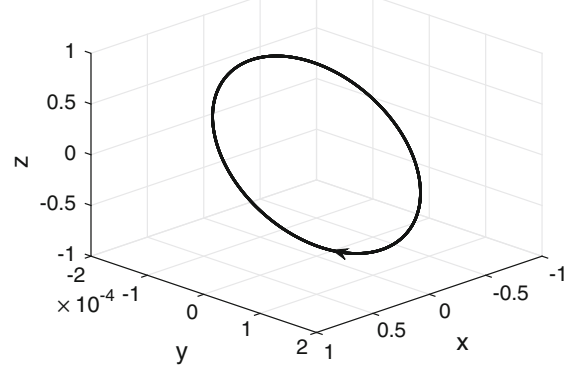

(a)

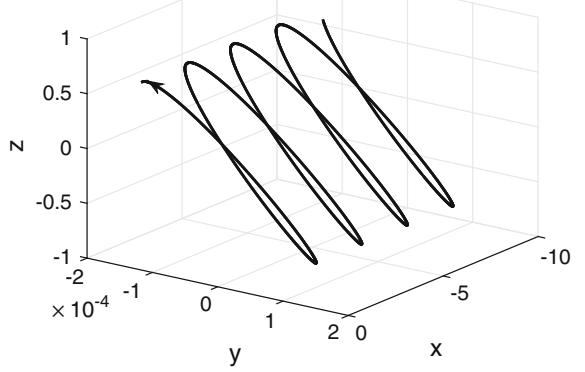

(b)

Fig. 1 Particle paths on the free surface at a latitude $\phi=60^{\circ} \mathrm{N}$ for $a=2 \mathrm{~m}$ and $L=100 \mathrm{~m}$. a $c_{0}=0 \mathrm{~ms}^{-1}$, b $c_{0}=0.3 \mathrm{~ms}^{-1}$

certain relations allowing the existence of such exact and physically plausible solution, or more precisely allowing the existence of a continuous pressure compatible with (2.1) and (2.2). The complete analysis and determination of the conditions on those parameters is given in full detail in [9]; however, in order to be self-contained, we indicate here the most relevant relations for our purposes. Firstly, the boundary condition (2.1d) is responsible for the following relation

$$
b^{2}=a^{2}+d^{2} .
$$

Secondly, in order to maintain the mass conservation (or equivalently, a volume-preserving situation), the Jacobian of the solution (2.2) in terms of the labelling variables must be independent of time. Specifically, this Jacobian is given by

$$
(a m-b k) e^{m s} \cos [k(q-c t)]+1-a b m k e^{2 m s}
$$

and therefore, the following relation must hold,

$$
a m=b k .
$$

Finally, the following dispersion relation is derived,

$$
\left[k^{2} c^{3}-f^{2}\left(c-c_{0}\right)\right]^{2}=\left(k^{2} c^{2}-f^{2}\right)\left[g-\hat{f}\left(c-c_{0}\right)\right]^{2}
$$

Furthermore, also from [9],

$$
m \geq k \text {. }
$$

In particular, for midlatitudes an approximation of the parameter $m$ is obtained as follows

$$
\begin{aligned}
& |m| \approx \frac{g}{f c_{0}} k, \quad \text { when } c_{0} \neq 0, \\
& |m| \approx \frac{g}{g-\hat{f} c} k, \quad \text { when } c_{0}=0,
\end{aligned}
$$

where both $\frac{g}{f c_{0}}$ and $\frac{g}{g-\hat{f} c}$ are greater than 1 , so indeed $m>k>0$. Lastly, we present an example of the free surface (see Fig.2), which prescribes the wave shape and whose determination is one of the main factors making the general water wave problem so complex. For this particular case, the free surface can be obtained explicitly as a parametric surface (with parameters $q$ and $r$ ) by taking advantage of the Lagrangian description (2.2) and the boundary conditions on the pressure. 


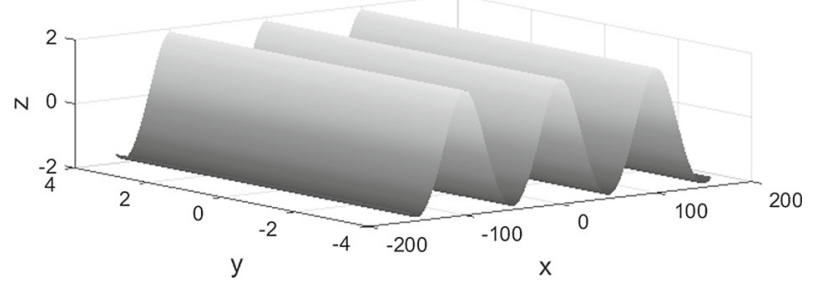

Fig. 2 Free surface at the equator $(\phi=0)$ for $a=10 \mathrm{~m}$ and $L=100 \mathrm{~m}$

The infinite fluid region to be considered has a free surface like the one in Fig. 2 as its upper boundary.

\section{Main results}

In order to rigorously show that the fluid motion given by (2.2) is dynamically possible, in addition to deriving a suitable pressure (as it was already done in [9]), we need to show that the solution describes a motion of the whole fluid body located below the free surface and where there are not particles colliding. This idea can be expressed in mathematical terms as the following statement; for each given time $t \geq 0$, the map given by (2.2) has to be a global diffeomorphism from the labelling domain into the fluid domain. This section is devoted to prove the previous statement, which is summarised in Theorem 3.1.

Firstly, we note that the case of an arbitrary time $t \geq 0$ follows from the case $t=0$, for which (2.2) takes the form

$$
\left\{\begin{array}{l}
x=q-b e^{m s} \sin (k q) \\
y=r-d e^{m s} \cos (k q) . \\
z=s+a e^{m s} \cos (k q)
\end{array}\right.
$$

If the following notation is introduced,

$$
(q, r, s) \in \mathscr{D}_{0} \longmapsto \mathscr{F}(q, r, s)=\left[\begin{array}{c}
q-b e^{m s} \sin (k q) \\
r-d e^{m s} \cos (k q) \\
s+a e^{m s} \cos (k q)
\end{array}\right]
$$

where

$$
\mathscr{D}_{0}=\left\{(q, r, s): q \in \mathbb{R}, r \in\left(-r_{0}, r_{0}\right), s \in\left(-\infty, s_{0}(r)\right)\right\},
$$

then, (2.2) can be recovered by considering the following change of variables and shift in the $x$ component

$$
\mathscr{F}(q-c t, r, s)+\left[\begin{array}{l}
\left(c-c_{0}\right) t \\
0 \\
0
\end{array}\right] .
$$

Furthermore, the map (3.1) is periodic in $q$ with period $2 \pi / k$ for both, the $y$ and the $z$ component, and "almost" periodic in the $x$ component, for which it experiences a shift of $2 \pi / k$ that does not affect the diffeomorphic character of the map in the whole real line. Therefore, showing that (2.2) is a global diffeomorphism in $\mathscr{D}_{0}$, it is reduced to proving that the map $\mathscr{F}$ is a global diffeomorphism from the domain

$$
\mathscr{D}=\left\{(q, r, s): q \in\left(0, \frac{2 \pi}{k}\right), r \in\left(-r_{0}, r_{0}\right), s \in\left(-\infty, s_{0}(r)\right)\right\} .
$$


into the fluid domain below the free surface. Thus, the main result of this paper is stated in the following way.

Theorem 3.1 If bme $^{m s_{0}(r)}<1$ then the map $\mathscr{F}$ is a global diffeomorphism from $\mathscr{D}$ into the fluid region below the free surface. Therefore, the Lagrangian flow-map (2.2) is a global diffeomorphism from $\mathscr{D}_{0}$ into the fluid domain.

The proof of this result follows the approach initiated in [13] and continued in [28,30], where the use of the Invariance of Domain theorem has proven to be an elegant tool which simplifies the direct calculations done in the first-known rigorous analysis of Gerstner wave solution [3]. Before proving (3.1), we present and discuss some results. The first proposition, already outlined in [9], establishes a first result regarding the regularity of the map $\mathscr{F}$.

Proposition 3.1 Under the condition $1-a m e^{m s_{0}(r)}>0$, the map $\mathscr{F}$ is a local diffeomorphism from $\mathscr{D}$ into its image, the fluid region below the free surface.

Proof The Jacobian matrix of $\mathscr{F}$ is given by

$$
D \mathscr{F}_{(q, r, s)}=\left(\begin{array}{lll}
1-b k e^{m s} \cos (k q) & 0 & -b m e^{m s} \sin (k q) \\
d k e^{m s} \sin (k q) & 1 & -d m e^{m s} \cos (k q) \\
-a k e^{m s} \sin (k q) & 0 & 1+a m e^{m s} \cos (k q)
\end{array}\right)
$$

and its determinant is given by

$$
\left|\begin{array}{ll}
1-b k e^{m s} \cos (k q) & -b m e^{m s} \sin (k q) \\
-a k e^{m s} \sin (k q) & 1+a m e^{m s} \cos (k q)
\end{array}\right|=1-a b m k e^{2 m s}+(a m-b k) e^{m s} \cos (k q) .
$$

From (2.4), it follows that the determinant is reduced to,

$$
1-a^{2} m^{2} e^{2 m s} \text {. }
$$

Thus, under the hypothesis that $1-a m e^{m s_{0}(r)}>0$, the determinant of the Jacobian matrix is nonzero for all $s \in\left(-\infty, s_{0}(r)\right)$. On the other hand, $\mathscr{F}$ has continuous partial derivatives in any neighbourhood contained in $\mathscr{D}$. Hence, $\mathscr{F}$ is a continuously differentiable map such that its Jacobian is strictly positive, so $\mathscr{F}$ is a local diffeomorphism from $\mathscr{D}$ into its range due to the Inverse Function Theorem.

Let us now provide conditions under which the map $\mathscr{F}$ is globally injective.

\section{Proposition 3.2 If}

$$
b m e^{m s_{0}(r)}<1
$$

then, $\mathscr{F}$ is globally injective on $\mathscr{D}$.

Proof Let us write $\mathscr{F}$ in the following form

$$
\mathscr{F}(q, r, s)=(q, r, s)+\mathscr{G}(q, r, s)
$$

where

$$
\mathscr{G}(q, r, s)=-e^{m s}(b \sin (k q), d \cos (k q),-a \cos (k q))
$$

We note now that $\mathscr{G}$ is a continuously differentiable map and it satisfies the following mean value theorem type of inequality

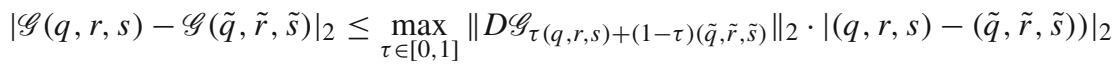




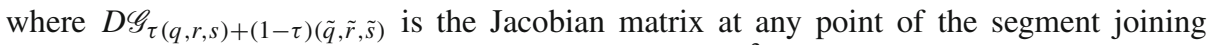
$(q, r, s)$ and $(\tilde{q}, \tilde{r}, \tilde{s}),|\cdot|_{2}$ is the Euclidean norm in $\mathbb{R}^{3}$, and $\|\cdot\|_{2}$ is the operator norm induced by the previous norm $|\cdot|_{2}$, i.e. given an arbitrary three-by-three matrix $M$,

$$
\|M\|_{2}=\sup \left\{|M(q, r, s)|_{2}:(q, r, s) \in \mathbb{R}^{3} \text { such that }|(q, r, s)|_{2}=1\right\}
$$

For this particular case, the matrix norm $\|\cdot\|_{2}$ is equivalent to the so-called spectral matrix norm [18]. The spectral norm of a matrix $M$ is the square root of the largest eigenvalue of the positive-semidefinite matrix $M^{*} M$,

$$
\|M\|_{2}=\sqrt{\lambda_{\max }\left(M^{*} M\right)}
$$

where $M^{*}$ is the conjugate transpose of $M$. Let first obtain the Jacobian matrix of $\mathscr{G}$,

$$
D \mathscr{G}_{(q, r, s)}=\left(\begin{array}{lll}
-b k e^{m s} \cos (k q) & 0 & -b m e^{m s} \sin (k q) \\
d k e^{m s} \sin (k q) & 0 & -d m e^{m s} \cos (k q) \\
-a k e^{m s} \sin (k q) & 0 & a m e^{m s} \cos (k q)
\end{array}\right) .
$$

Now, for this real matrix, $D \mathscr{G}_{(q, r, s)}^{*} D \mathscr{G}_{(q, r, s)}$ is given by

$$
\begin{aligned}
& e^{2 m s}\left(\begin{array}{lll}
b^{2} k^{2} \cos ^{2}(k q)+\left(d^{2}+a^{2}\right) k^{2} \sin ^{2}(k q) & 0 & \left(b^{2}-d^{2}-a^{2}\right) k m \sin (k q) \cos (k q) \\
0 & 0 & 0 \\
\left(b^{2}-d^{2}-a^{2}\right) k m \sin (k q) \cos (k q) & 0 & b^{2} m^{2} \sin ^{2}(k q)+\left(d^{2}+a^{2}\right) m^{2} \cos ^{2}(k q)
\end{array}\right) \\
& =e^{2 m s}\left(\begin{array}{lll}
b^{2} k^{2} & 0 & 0 \\
0 & 0 & 0 \\
0 & 0 & b^{2} m^{2}
\end{array}\right) \\
& =b^{2} e^{2 m s}\left(\begin{array}{lll}
k^{2} & 0 & 0 \\
0 & 0 & 0 \\
0 & 0 & m^{2}
\end{array}\right),
\end{aligned}
$$

where the second equality follows from (2.3). Hence, $\left\{0, b^{2} k^{2} e^{2 m s}, b^{2} m^{2} e^{2 m s}\right\}$ is the set of eigenvalues of $D \mathscr{G}_{(q, r, s)}^{T} D \mathscr{G}_{(q, r, s)}$. The maximum of this set is readily obtained from the inequality (2.6). Consequently,

$$
\left\|D \mathscr{G}_{(q, r, s)}\right\|_{2}=\sqrt{b^{2} m^{2} e^{2 m s}}=b m e^{m s}
$$

On the other hand,

$$
\max _{\tau \in[0,1]}\left\|D \mathscr{G}_{\tau(q, r, s)+(1-\tau)(\tilde{q}, \tilde{r}, \tilde{s})}\right\|_{2}=b m e^{m s^{*}}
$$

where $s^{*}$ is the $s$-component of the point on the line segment joining $(q, r, s)$ and $(\tilde{q}, \tilde{r}, \tilde{s})$ that maximises $b m e^{m s}$. In particular, $s^{*} \leq s_{0}(r)$. Now by (3.6),

$$
\left.|\mathscr{G}(q, r, s)-\mathscr{G}(\tilde{q}, \tilde{r}, \tilde{s})|_{2} \leq b m e^{m s^{*}} \mid(q, r, s)-(\tilde{q}, \tilde{r}, \tilde{s})\right)\left.\right|_{2} .
$$

Returning to the function $\mathscr{F}$, the previous inequality yields

$$
\begin{aligned}
|\mathscr{F}(q, r, s)-\mathscr{F}(\tilde{q}, \tilde{r}, \tilde{s})|_{2} & =|(q, r, s)-(\tilde{q}, \tilde{r}, \tilde{s})+\mathscr{G}(q, r, s)-\mathscr{G}(\tilde{q}, \tilde{r}, \tilde{s})|_{2} \\
& =|(q, r, s)-(\tilde{q}, \tilde{r}, \tilde{s})-(\mathscr{G}(\tilde{q}, \tilde{r}, \tilde{s})-\mathscr{G}(q, r, s))|_{2} \\
& \left.\geq|(q, r, s)-(\tilde{q}, \tilde{r}, \tilde{s})|_{2}-\mid \mathscr{G}(q, r, s)\right)-\left.\mathscr{G}(\tilde{q}, \tilde{r}, \tilde{s})\right|_{2} \\
& \geq|(q, r, s)-(\tilde{q}, \tilde{r}, \tilde{s})|_{2}-b m e^{m s^{*}}|(q, r, s)-(\tilde{q}, \tilde{r}, \tilde{s})|_{2} \\
& =\left(1-b m e^{m s^{*}}\right)|(q, r, s)-(\tilde{q}, \tilde{r}, \tilde{s})|_{2}
\end{aligned}
$$


Finally, by the hypothesis (3.5), $1-b m e^{m s^{*}} \geq 1-b m e^{m s_{0}(r)}>0$, so

$$
|(q, r, s)-(\tilde{q}, \tilde{r}, \tilde{s})|_{2} \leq \frac{1}{1-b m e^{m s^{*}}}|\mathscr{F}(q, r, s)-\mathscr{F}(\tilde{q}, \tilde{r}, \tilde{s})|_{2}
$$

which, by means of the properties of the norm, proves that the map $\mathscr{F}$ is globally injective.

In order to completely characterise the injectivity of $\mathscr{F}$, we obtain the following necessary condition.

Lemma 3.1 If $\mathscr{F}$ is injective, then it follows that

$$
b k e^{m s_{0}(r)} \leq 1
$$

Proof Let us consider a family of nonlinear equations in the variable $q$ given by

$$
\mathscr{H}(q):=q-b e^{m s} \sin (k q)=0
$$

We prove that the former equation has more than one solution. Let us assume that (3.9) does not hold, i.e.

$$
b k e^{m s_{0}(r)}>1 .
$$

We obtain that $\mathscr{H}^{\prime}(q)=0$ if and only if

$$
q=\frac{1}{k} \arccos \left(\frac{1}{b k e^{m s}}\right)
$$

which by (3.11) is well-defined. Indeed, it is possible to find $q_{0} \in\left(0, \frac{\pi}{2 k}\right)$ such that $q_{0}$ is a minimum for $\mathscr{H}$ and such that $\mathscr{H}\left(q_{0}\right)<0$. From the fact that $\mathscr{H}(0)=0$, it follows that there exists a strictly positive solution of (3.10). If $\alpha$ is such solution, then it readily follows that $-\alpha$ is also a solution. Hence, the mapping $\mathscr{F}$ is not injective, as the points

$$
\begin{aligned}
& \left(q_{1}, r_{1}, s_{1}\right)=(\alpha, r, s) \\
& \left(q_{2}, r_{2}, s_{2}\right)=(-\alpha, r, s)
\end{aligned}
$$

are such that

$$
\mathscr{F}\left(q_{1}, r_{1}, s_{1}\right)=\mathscr{F}\left(q_{2}, r_{2}, s_{2}\right)
$$

Remark 3.1 We establish here the relation between the assumptions considered in Propositions 3.1 and 3.2. If we take into account that both $a$ and $b$ are positive, together with the condition (2.3) it follows that

$$
b \geq a
$$

which yields

$$
1-b m e^{m s} \leq 1-a m e^{m s}, \text { for all } m>0 .
$$

Therefore, the condition (3.5) for the global injectivity of $\mathscr{F}$ implies the condition for the local diffeomorphic character of $\mathscr{F}$. In particular, along the Equator $a=b$ and $m=k$ (by (2.4)). Therefore, the necessary condition for injectivity (3.9) and the sufficient condition (3.5) coincide for all smooth free surfaces (as equality in (3.9) implies that the free surface has the shape of an inverted cycloid), as well as the condition establishing the diffeomorphic character of $\mathscr{F}$ (see Proposition 3.1). On the other hand, outside the Equator $m$ is strictly greater than $k$, and therefore the condition for global injectivity (3.5) is stronger than the 
condition assuring the local diffeomorphic character of $\mathscr{F}$. In this case, we might have a set of values for the parameters $a, b, m$, and $k$ producing a solution to the governing equations (2.1) but such that $1>a m e^{m s_{0}(r)}$ whereas $1<b m e^{m s_{0}(r)}$; therefore, enabling $\mathscr{F}$ to be a local diffeomorphism which is not globally injective.

We state now a remarkable theorem in topology and analysis whose proof in a general setting can be found in [29].

Theorem (Invariance of Domain Theorem) If $U \subset \mathbb{R}^{n}$ is an open set and $F: \bar{U} \rightarrow \mathbb{R}^{n}$ is a continuous one-to-one mapping, then $F: U \rightarrow F(U)$ is a homeomorphism, and $F(\partial U)=\partial F(U)$.

The power of the previous theorem is readily seen in the proof of the main result of the present paper.

Proof of Theorem 3.1 It has been proven in Proposition 3.2 that, when the hypothesis in Theorem 3.1 holds, i.e. when

$$
b m e^{m s_{0}(r)}<1,
$$

the map $\mathscr{F}$ is globally injective. In addition, one can readily check the continuity of $\mathscr{F}$ in the open set $\mathscr{D}_{0}$. Therefore, by the invariance of domain theorem, $\mathscr{F}$ is a homeomorphism that maps the image of the boundary of $\mathscr{D}_{0}$ into the boundary of the image $\mathscr{F}\left(\mathscr{D}_{0}\right)$, which constitutes the free surface. In particular, $\mathscr{F}$ is globally bijective. On the other hand, the condition (3.12) implies that

$$
a m e^{m s_{0}(r)}<1 .
$$

Hence, the hypothesis in Proposition 3.1 is satisfied and therefore $\mathscr{F}$ is a local diffeomorphism. Finally, we have that $\mathscr{F}$ is a local diffeomorphism which is globally bijective, therefore $\mathscr{F}$ is a global diffeomorphism.

Acknowledgements The author acknowledges the support of the Science Foundation Ireland (SFI) research Grant 13/CDA/2117

\section{A Appendix: mean value theorem inequality}

Let us first define the norms that will be used in the following result.

Definition A.1 The norm 2 of a vector $\mathbf{x}=\left[x_{1}, \ldots, x_{n}\right]$ is given by

$$
|\mathbf{x}|_{2}=\sqrt{\sum_{k=1}^{n}\left|x_{k}\right|^{2}}
$$

Definition A.2 The matrix norm induced by the previous norm is defined as

$$
\|M\|=\sup \left\{|M \mathbf{x}|_{2}: \mathbf{x} \in \mathbb{R}^{n} \text { such that }|\mathbf{x}|_{2}=1\right\}
$$

for an arbitrary $n \times n$ matrix $M$ (in general $M$ can be a non-square matrix).

The following result has been generalised to locally convex topological vector spaces by the use of the Gâteaux differential [1]. However, the proof for the case we are interested in can be simply obtained in the following manner. 
Theorem A.1 Let $f: O \subset \mathbb{R}^{n} \longrightarrow \mathbb{R}^{n}$ be a differentiable vector-valued function on a convex open domain $O$. Then,

$$
|f(\mathbf{y})-f(\mathbf{x})|_{2} \leq \sup _{\mathbf{z} \in\{\mathbf{x}, \mathbf{y}\}}\left\|D f_{\mathbf{z}}\right\| \cdot|\mathbf{y}-\mathbf{x}|_{2}
$$

where $\{\mathbf{x}, \mathbf{y}\}$ represents the segment joining $\mathbf{x}$ and $\mathbf{y}$, and $D f_{\mathbf{z}}$ is the Jacobian matrix of $f$ at z.

Proof Let us define the function $\varphi:[0,1] \longrightarrow \mathbb{R}$

$$
\varphi(t)=\langle f(\mathbf{y})-f(\mathbf{x}), f(\mathbf{x}+t(\mathbf{y}-\mathbf{x}))\rangle
$$

which is well-defined because $O$ is a convex domain. In particular,

$$
\varphi(1)=\langle f(\mathbf{y})-f(\mathbf{x}), f(\mathbf{y})\rangle
$$

and

$$
\varphi(0)=\langle f(\mathbf{y})-f(\mathbf{x}), f(\mathbf{x})\rangle
$$

Due to the regularity of the inner product $\langle\cdot, \cdot\rangle$ and the function $f$, we can apply the mean value theorem to $\varphi$. Thus, there exists $\tau \in(0,1)$ such that

$$
\varphi(1)-\varphi(0)=\varphi^{\prime}(\tau)
$$

We have that $\mathbb{R}^{n}$ is a Hilbert space with the norm 2 (A.1) and therefore,

$$
\begin{aligned}
\varphi(1)-\varphi(0) & =\langle f(\mathbf{y})-f(\mathbf{x}), f(\mathbf{y})\rangle-\langle f(\mathbf{y})-f(\mathbf{x}), f(\mathbf{x})\rangle \\
& =\langle f(\mathbf{y})-f(\mathbf{x}), f(\mathbf{y})-f(\mathbf{x})\rangle \\
& =|f(\mathbf{y})-f(\mathbf{x})|_{2}^{2}
\end{aligned}
$$

On the other hand, the matrix norm (A.2) satisfies that $|M \mathbf{x}| \leq\|M\||\mathbf{x}|$, hence

$$
\begin{aligned}
\varphi^{\prime}(\tau) & =\left\langle f(\mathbf{y})-f(\mathbf{x}), D f_{\mathbf{x}+\tau(\mathbf{y}-\mathbf{x})}[\mathbf{y}-\mathbf{x}]\right\rangle \\
& \leq|f(\mathbf{y})-f(\mathbf{x})|_{2}\left|D f_{\mathbf{x}+\tau(\mathbf{y}-\mathbf{x})}[\mathbf{y}-\mathbf{x}]\right|_{2} \\
& \leq|f(\mathbf{y})-f(\mathbf{x})|_{2}\left(\left\|D f_{\mathbf{x}+\tau(\mathbf{y}-\mathbf{x})}\right\||\mathbf{y}-\mathbf{x}|_{2}\right) \\
& \leq|f(\mathbf{y})-f(\mathbf{x})|_{2}\left(\sup _{t \in(0,1)}\left\|D f_{\mathbf{x}+t(\mathbf{y}-\mathbf{x})}\right\||\mathbf{y}-\mathbf{x}|_{2}\right) \\
& =|f(\mathbf{y})-f(\mathbf{x})|_{2}\left(\sup _{\mathbf{z} \in\{x, y\}}\left\|D f_{\mathbf{z}}\right\||\mathbf{y}-\mathbf{x}|_{2}\right)
\end{aligned}
$$

where $\mathbf{z}=\mathbf{x}+t(\mathbf{y}-\mathbf{x})$ is any point on the segment $\{\mathbf{x}, \mathbf{y}\}$. Therefore, by (1.5),

$$
|f(\mathbf{y})-f(\mathbf{x})|_{2}^{2} \leq|f(\mathbf{y})-f(\mathbf{x})|_{2}\left(\sup _{\mathbf{z} \in\{x, y\}}\left\|D f_{\mathbf{z}}\right\||\mathbf{y}-\mathbf{x}|_{2}\right)
$$

Finally, if $f(\mathbf{x})=f(\mathbf{y})$ then (1.3) follows trivially; whereas if $f(\mathbf{x}) \neq f(\mathbf{y})$, we can divide (1.6) by $|f(\mathbf{y})-f(\mathbf{x})|_{2}$ which yields the desired result (1.3). 


\section{References}

1. Ambrosetti, A., Prodi, G.: A Primer of Nonlinear Analysis. Cambridge University Press, Cambridge (1993)

2. Bennett, A.: Lagrangian Fluid Dynamics. Cambridge University Press, Cambridge (2006)

3. Constantin, A.: On the deep water wave motion. J. Phys. A: Math. Gen. 34(7), 1405-1417 (2001a)

4. Constantin, A.: Edge waves along a sloping bed. J. Phys. A 34(45), 9723-9731 (2001b)

5. Constantin, A.: Nonlinear Water Waves with Applications to Wave-Current Interactions and Tsunamis, CBMS-NSF Conference Series in Applied Mathematics, vol. 81. SIAM, Philadelphia (2011)

6. Constantin, A.: An exact solution for equatorially trapped waves. J. Geophys. Res. 117, C05029 (2012). https://doi.org/10.1029/2012JC007879

7. Constantin, A.: Some three-dimensional nonlinear equatorial flows. J. Phys. Oceanogr. 43, 165-175 (2013)

8. Constantin, A.: Some nonlinear, equatorially trapped, nonhydrostatic internal geophysical waves. J. Phys. Oceanogr. 44, 781-789 (2014)

9. Constantin, A., Monismith, S.G.: Gerstner waves in the presence of mean currents and rotation. J. Fluid. Mech. 820, 511-528 (2017). https://doi.org/10.1017/jfm.2017.223

10. Cushman-Roisin, B., Beckers, J.M.: Introduction to Geophysical Fluid Dynamics: Physical and Numerical Aspects, vol. 101. Academic Press, Cambridge (2011)

11. Froude, W.: On the rolling of ships. Trans. Inst. Naval Arch. 3, 45-62 (1862)

12. Gerstner, F.: Theorie der Wellen samt einer daraus abgeleiteten Theorie der Deichprofile. Ann. Phys. 2, 412-445 (1809)

13. Henry, D.: On Gerstner's water wave. J. Nonlinear Math. Phys. 15(sup2), 87-95 (2008)

14. Henry, D.: An exact solution for Equatorial geophysical water waves with an underlying current. Eur. J. Mech. B. Fluids 38, 190-195 (2013)

15. Henry, D.: Equatorially trapped nonlinear water waves in a $\beta$-plane approximation with centripetal forces. J. Fluid Mech. 804, R1 (2016). https://doi.org/10.1017/jfm.2016.544

16. Henry, D.: A modified equatorial $\beta$-plane approximation modelling nonlinear wave-current interactions. J. Differ. Equ. 263, 2554-2566 (2017)

17. Henry, D.: On three-dimensional Gerstner-like equatorial water waves. Philos. Trans. R. Soc. A. Math Phys. Eng Sci. 376(2111), 20170088 (2018). https://doi.org/10.1098/rsta.2017.0088

18. Horn, R.A., Johnson, C.R.: Matrix Analysis, p. 346. Cambridge University Press, Cambridge (1985)

19. Hsu, H.-C.: An exact solution for nonlinear internal Equatorial waves in the $f$-plane approximation. J. Math. Fluid Mech. 16, 463-471 (2014)

20. Ionescu-Kruse, D.: An exact solution for geophysical edge waves in the $f$-plane approximation. Nonlinear Anal. Real World Appl. 24, 190-195 (2015)

21. Johnson, R.S.: Application of the ideas and techniques of classical fluid mechanics to some problems in physical oceanography. Philos. Trans. R. Soc. A. Math Phys. Eng Sci. 376(2111), 20170092 (2018). https://doi.org/10.1098/rsta.2017.0092

22. Kluczek, M.: Exact and explicit internal Equatorially-trapped water waves with underlying currents. J. Math. Fluid Mech. 10(2), 305-314 (2016). https://doi.org/10.1007/s00021-016-0281-6

23. Monismith, S., Nepf, H., Cowen, E., Thais, L., Magnaudet, J.: Laboratory observations of mean flows under surface gravity waves. J. Fluid Mech. 573, 131-147 (2007)

24. Matioc, A.V.: Exact geophysical waves in stratified fluids. Appl. Anal. 92, 2254-2261 (2013)

25. Pollard, R.T.: Surface waves with rotation: an exact solution. J. Geophys. Res. 75, 5895-5898 (1970)

26. Rankine, W.J.M.: On the exact form of waves near the surface of deep water. Phil. Trans. R. Soc. Lond. A 153, 127-138 (1863)

27. Reech, F.: Sur la théorie des ondes liquides périodiques. C. R. Acad. Sci. Paris 68, 1099-1101

28. Rodríguez-Sanjurjo, A.: Global diffeomorphism of the Lagrangian flow-map for Equatorially trapped internal water waves. Nonlinear Anal. 149, 156-164 (2017)

29. Rothe, E.H.: Introduction to Various Aspects of Degree Theory in Banach Spaces. Mathematical Surveys and Monographs, vol. 23. American Mathematical Society, Providence (1986)

30. Sastre-Gomez, S.: Global diffeomorphism of the Lagrangian flow-map defining equatorially trapped water waves. Nonlinear Anal. 125, 725-731 (2015)

31. Stuhlmeier, R.: Internal Gerstner waves: applications to dead water. Appl. Anal. 93, 1451-1457 (2014) 